\title{
Control of optical bistability and complex dynamics of a nonlinear interferometer
}

\author{
I.N. Agishev, N.A. Ivanova, A.L. Tolstik \\ Department of Laser Physics and Spectroscopy, Belarussian State University, 4 F. Skaryna Ave., Minsk 220050, Belarus
}

Received 16 April 1998; accepted 10 June 1998

\begin{abstract}
Transfer functions and dynamic characteristics of a Fabry-Perot interferometer with a multilevel resonant medium have been studied theoretically under conditions of two-frequency excitation when one of the light beams had no resonator feedback. An incoherent control method, using absorption of an independent light beam by molecules in excited energy states, has been proposed for the bistable and dynamic characteristics of a nonlinear Fabry-Perot interferometer. (C) 1998 Elsevier Science B.V. All rights reserved.
\end{abstract}

\section{Introduction}

As a consequence of the development of an elemental base for optical data-processing systems, much attention has been paid to the interaction of several light beams with a nonlinear Fabry-Perot interferometer (FPI). Based on a nonlinear FPI, an analogue of an electronic transistor (transphasor), optical bistability, i.e. the generation modes of intensity pulsations at the output of the interferometer with fixed input intensity have been realized. Nonlinear interferometers provide an excellent example of nonlinear systems with optical feedback, in which various effects of spatio-temporal self-organization appear [1]. The use of an additional light beam in the interferometer makes it possible to realize the control of functional characteristics for nonlinear systems. It has been proposed to use light fields, differing in frequency [2,3] or directed to the interferometer input at different angles [4], with the aim of realizing switchable optical devices. The possibility of generating intensity oscillations in the interferometer through the concurrence of two nonlinearity mechanisms of the refractive index with different relaxation times has been demonstrated in Ref. [5]. In an effort to produce high-frequency oscillations, it has been proposed [6,7] to use two-component or multilevel media, characterized by nonmonotonic dependence of the refractive index on the intensity due to population of different molecular energy states [8].

This paper presents a theoretical analysis of the control method for optical bistability and dynamic operation modes of a Fabry-Perot interferometer based on the change in the nonlinear properties of multilevel resonant media upon absorption of an independent light beam (optical pumping) from the excited level. Switching of resonance and/or thermal nonlinearity in the excited channel results in changing of the medium properties, influencing the character of the interaction between a signal wave and the nonlinear interferometer. As this takes place, a means is provided for amplification of the medium nonlinear response induced by a weak signal beam.

\section{Optical bistability and control over the transfer function of a nonlinear interferometer}

Consideration is being given to a model of a Fabry-Perot interferometer with a multilevel resonant medium taking into account the transitions to excited singlet $S_{1}, S_{2}$ and triplet $T_{1}, T_{2}$ states of the molecules. The levels shown schematically in Fig. 1 represent the patterns of energy states characteristic for complex organic compounds and may be used to describe 
a)
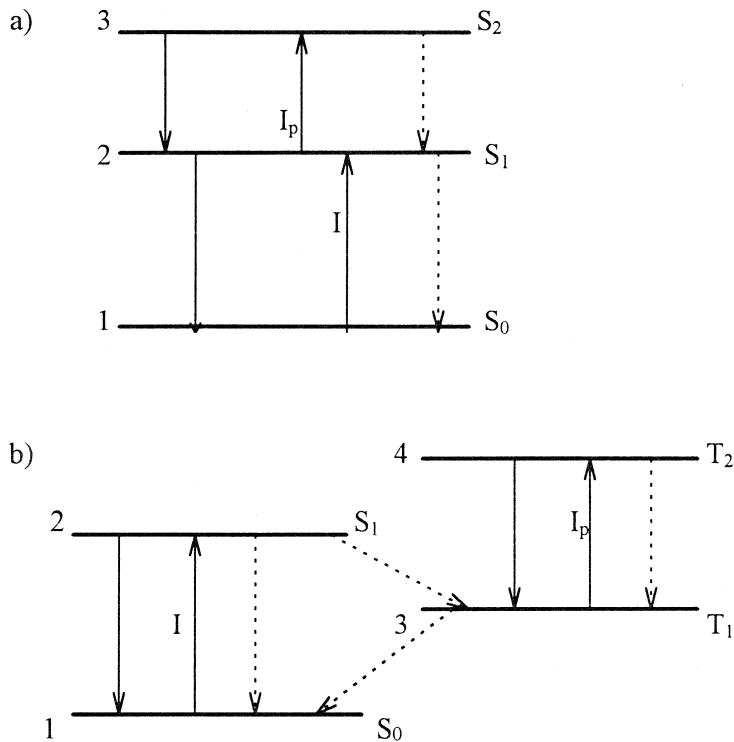

c)

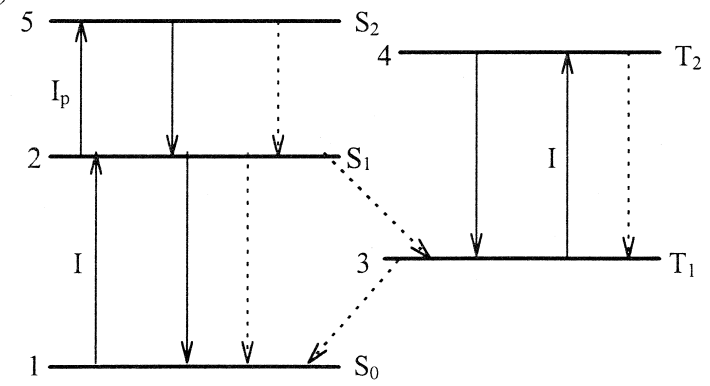

Fig. 1. Position of the molecular energy states modelled by three-, four- and five-level schemes. Arrows denote stimulated (solid lines), spontaneous and radiationless (dashed lines) transitions.

liquid and solid dye solutions, molecular crystals, colored crystals, and vapors of complex organic compounds. In doing so, one has the possibility to describe the nonlinear optical characteristics of the medium with any relative positions of the absorption (emission) bands from ground and excited levels for arbitrary probability values of spontaneous and radiationless transitions [8].

We analyze the FPI transfer function in case of two-frequency excitation. Let the signal beam $I$ be tuned into the absorption band of the principal channel $S_{0}-S_{1}$ and have no direct interaction with the excited channel $S_{1}-S_{2}\left(T_{1}-T_{2}\right)$. The frequency of optical pumping $I_{\mathrm{p}}$ lies within the region of the shifted absorption band of the excited singlet or triplet level (Figs. 1a, 1b). In this case it is assumed that the resonator feedback is available for a signal wave only, and optical pumping may have any propagation direction. Because of this, at minor absorption of optical pumping in the excited channel under conditions of single-pass radiation propagation through the medium the pumping intensity may be thought of as constant for any medium region.

The relation between the averaged field intensity in the medium $I$ and the intensity of the light beam $I_{0}$ incident at FPI for a signal light beam can be represented as [9]:

$$
I=\frac{I_{0}(1-R)(1-\tau)(1+R \tau)}{k L\left[(1-R \tau)^{2}+4 R \tau \sin ^{2}(\Phi)\right]},
$$

where $\tau=\exp (-k L)$ is the transmission of a layer with thickness $L, R$ is the reflection coefficient of the mirrors, $\Phi=2 \pi n L / \lambda-m \pi$ is the phase detuning of FPI out of resonance, $n$ is the medium refractive index, $\lambda$ is the radiation wavelength, and $m$ is an integer number. 
The intensity of the transmitted light flux is given as follows:

$$
I_{\text {out }}=\frac{k L}{1-\tau} \frac{I(1-R) \tau}{1+R \tau} .
$$

With the aim of calculating the nonlinear properties of the resonant medium, we use the standard solution procedure of the kinetic equations for the level populations and the Kramers-Kronig relations relating the real and imaginary parts of the complex refractive index $\hat{n}=n+\mathrm{i} \varkappa$ (dispersion relations, see, e.g., Refs. [10,11]). This approach allows for molecular transitions to the excited state and the Stokes shift of absorption and emission bands associated with complex organic molecules [8]. For media modelled by three- and four-level schemes (Figs. 1a, 1b) the complex refractive index of the medium in the field of two monochromatic light beams with intensities $I$ (signal wave) and $I_{\mathrm{p}}$ (optical pumping) may be represented as [12]:

$$
\hat{n}=n+\mathrm{i} \varkappa=n_{0}+\varkappa_{0}\left(\frac{\hat{\Theta}_{12}}{B_{12}}-\frac{\hat{\alpha} I}{1+\alpha I}\right),
$$

where $n_{0}$ is the nonresonant component of the refractive index (that is defined by the refractive index of a solvent or buffer gas), $\varkappa_{0}=c k_{0} / 2 \omega$ is a linear extinction coefficient, $k_{0}$ is the initial absorption coefficient. The complex nonlinearity parameter $\hat{\alpha}$, dependent on the spectral characteristics of the medium, pumping intensity and taking into account the absorbed energy thermalization in the principal $S_{0}-S_{1}$ and excited $S_{1}-S_{2}\left(T_{1}-T_{2}\right)$ channels, is determined by the following expressions:

$$
\hat{\alpha}=a+\mathrm{i} \alpha=\frac{\hat{\Theta}_{12}+\hat{\Theta}_{21}}{v P_{21}}-\sigma\left(1-\mu_{21}\right)+\frac{B_{23} I_{\mathrm{p}}\left(\hat{\Theta}_{12} / v P_{32}-\sigma_{\mathrm{p}}\left(1-\mu_{32}\right)\right)}{\left(1+B_{32} I_{\mathrm{p}} / v P_{32}\right) v P_{21}}
$$

for a three-level system, and

$$
\hat{\alpha}=\frac{\hat{\Theta}_{12}\left(1+P_{23} / P_{31}\right)+\hat{\Theta}_{21}}{v\left(P_{21}+P_{23}\right)}-\sigma\left(1-\mu_{21}\right)+\frac{B_{34} I_{\mathrm{p}}\left(\hat{\Theta}_{12} / v P_{43}-\sigma_{\mathrm{p}}\left(1-\mu_{43}\right)\right) P_{23}}{\left(1+B_{43} I_{\mathrm{p}} / v P_{43}\right) v\left(P_{21}+P_{23}\right) P_{31}}
$$

for the four-level one.

The complex parameter $\hat{\Theta}_{i j}=\Theta_{i j}+\mathrm{i} B_{i j}$ defines the spectral characteristics of the resonance transition $i-j$, and $\Theta_{i j}(\omega)$ is related to the Einstein coefficient for stimulated transitions $B_{i j}(\omega)$ through the dispersion relations: $\Theta_{i j}(\omega)=$ $(1 / \pi) \int_{-\infty}^{\infty} B_{i j}\left(\omega^{\prime}\right) /\left(\omega^{\prime}-\omega\right) \mathrm{d} \omega^{\prime}$ [11]. The Einstein coefficients $B_{12}$ and $B_{21}$ are determined at the frequency $\omega$ of a signal light beam, whereas $B_{23}\left(B_{34}\right)$ and $B_{32}\left(B_{43}\right)$ at the shifted frequency $\omega_{\mathrm{p}}$ of optical pumping $I_{\mathrm{p}}, v=c / n_{0}$ is the light velocity of the medium, $P_{i j}=A_{i j}+d_{i j}$ is the total probability of spontaneous $A_{i j}$ and radiationless $d_{i j}$ transitions in the $i-j$ channel, $\mu_{i j}=A_{i j} / P_{i j}$ is the quantum yield of luminescence, $\sigma_{(p)}=2 \omega_{(p)}(\mathrm{d} n / \mathrm{d} T) \delta t / c C_{\rho}, \mathrm{d} n / \mathrm{d} T$ is the thermooptic coefficient, $C_{\rho}$ is a unit-volume heat capacity, $\delta t$ is the characteristic duration of the interaction (upon pulse excitation when heat withdrawal processes could be neglected, $\delta t$ is the light pulse duration; under conditions of continuous laser excitation $\delta t$ is determined by the thermal relaxation time).

Numerical solution of coupled equations (1)-(3) has been obtained using the total intensity of a signal wave inside the interferometer $I$ as an independent variable. This solution allows for the determination of the refractive index $n$ and absorption coefficient $k=2 \omega \varkappa / c$ by Eq. (3), given the value of $I$, and for the determination of the input $I_{0}$ and output $I_{\text {out }}$ intensities of the interferometer by expressions (1), (2). Fig. 2 presents the intensity $I_{\text {out }}$ as a function of the input intensity $I_{0}$ for different values of optical pumping intensity $I_{\mathrm{p}}$ as applied to a three-level model assuming Gaussian approximation for absorption and luminescence bands and equal values of the Einstein coefficients at maxima of the profiles: $B_{12}^{m}=B_{21}^{m}=B_{23}^{m}$ $=B_{32}^{m}$. These functions have been obtained for the following parameters of the medium and radiation typical for the conditions of single-pulse excitation in ethanol solutions of dyes: excitation of the molecules is realized at maxima of the absorption bands $S_{0}-S_{1}$ and $S_{1}-S_{2} ; \lambda_{23}=2 \lambda_{12}=1 \mu \mathrm{m} ; \Delta_{23}=4 \Delta_{12}=60 \mathrm{~nm}\left(\lambda_{i j}\right.$ and $\Delta_{i j}$ are respectively the center and halfwidth of the absorption band in the $i-j$ channel); whereas the Stokes shift of absorption and emission bands in both spectral channels is equal to 0.4 of the profile halfwidth $\Delta_{12(23)}$; the quantum yield of luminescence in the spectral channel $S_{0}-S_{1}$ is $\mu_{21}=0.5 ; n_{0}=1.36 ;(\mathrm{d} n / \mathrm{d} T) C_{\rho}^{-1}=-2 \times 10^{-4} \mathrm{~J}^{-1} \mathrm{~cm}^{3}$ (using ethanol as a solvent); the characteristic duration of the interaction is $\delta t=10^{-8} \mathrm{~s}$; the optical density is $k_{0} L=0.02$; the reflection coefficient of the cavity mirrors is $R=0.99$; the initial detuning of the interferometer out of resonance $\Phi_{0}=2 \pi n_{0} L / \lambda-m \pi=0.03$. The intensities of the waves $I_{0}, I_{\text {out }}, I_{\mathrm{p}}$ are given with the normalization factor $B_{12}^{m} / v P_{21}$ determining the saturation intensity of the resonance transition $S_{0}-S_{1}\left(I_{S_{0}-S_{1}}=v P_{21} /\left(B_{12}+B_{21}\right)\right)$. The saturation intensity of the excited channel $I_{S_{1}--S_{2}}=100 I_{S_{0}--S_{1}}$ $\left(P_{32} / P_{21}=100\right)$. 


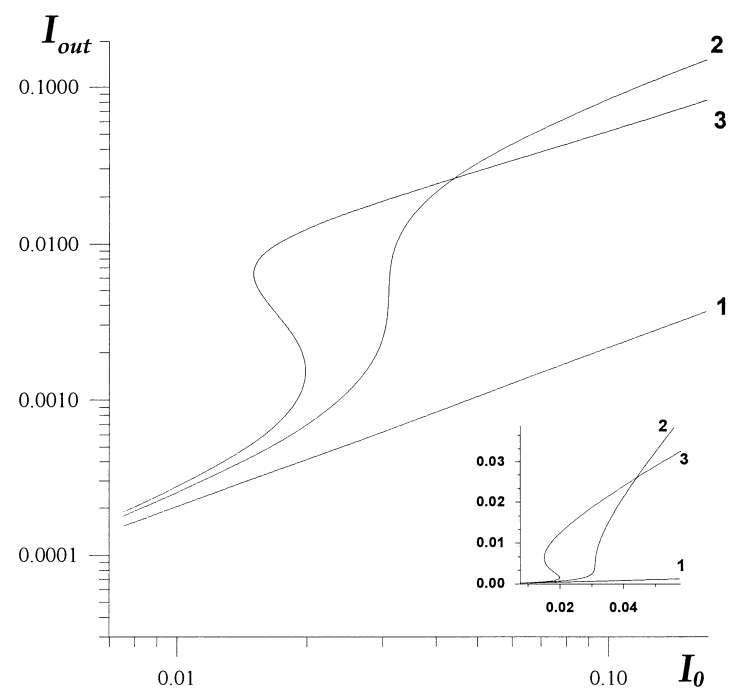

Fig. 2. Radiation intensity at the interferometer output $I_{\text {out }}$ as a function of the input intensity $I_{0}$ for different pumping intensities $I_{\mathrm{p}}=0(1)$, 1.7 (2), 4 (3).

As can be seen, the practically linear transmission characteristic (curve 1) is observed for the selected parameters of FPI without optical pumping. Absorption of optical pumping in the excited channel results in greater changes of the refractive index caused by a signal wave, leads to a change in the interferometer transfer function and provides a means for realization of FPI operating modes. In the mode of differential amplification (curve 2) it is possible to increase the small changes in the input intensity. With further increase in optical pumping one could realize optical bistability (curve 3 ). It should be noted that the optical bistability in the case illustrated in Fig. 2 has been obtained for $C=k_{0} L / 2(1-R)=1$, that is less than the values $C>4$ usually required for FPI [1].

In this way, switching-on of optical pumping makes it possible to realize the control over the interferometer transfer function, to change the differential amplification coefficient and to implement transition from mono to bistable state. Analogous functions have been also found for FPI with a resonant medium modelled by the four-level scheme when employing the pumping at the absorption frequency from the triplet level. It might be worthwhile to point out that allowance for thermal nonlinearity in the considered models of resonant media is not obligatory for the demonstration of the control effect over the FPI transfer functions. However, the inclusion of thermalization energy in the excited channel permits of a more spectacular illustration of the possibilities provided by additional nonlinearity upon absorption from the excited levels.

\section{Optical multistability}

More complex transfer functions of the interferometer are realized when excited singlet $S_{1}, S_{2}$ and triplet $T_{1}$, $T_{2}$ states are simultaneously involved in the interaction (five-level medium model, Fig. 1c). For the calculation of the nonlinear properties of such a five-level medium model it is assumed that a signal light beam falls within the bands of absorption from the ground singlet $S_{0}$ and triplet $T_{1}$ levels, and optical-pumping radiation is tuned into the band of absorption from the excited singlet level. Under these conditions the complex refractive index of the medium in a field of two monochromatic beams with different frequencies is determined by the following relation:

$$
\hat{n}=n+\mathrm{i} \varkappa=n_{0}+\varkappa_{0}\left(\frac{\hat{\Theta}_{12}}{B_{12}}-\frac{\hat{\alpha} I+\hat{\beta} I^{2}}{1+j I+\beta I^{2}}\right) .
$$

The parameters $j, \hat{\alpha}$ and $\hat{\beta}$ are defined by the spectral and thermooptical medium characteristics and may be represented as

$$
\begin{aligned}
& j=\frac{B_{12}\left(1+P_{23} / P_{31}\right)+B_{21}}{v\left(P_{21}+P_{23}\right)}+\frac{B_{43}}{v P_{43}}+\frac{B_{12} B_{25} I_{\mathrm{p}}}{\left(1+B_{52} I_{\mathrm{p}} / v P_{52}\right) v^{2}\left(P_{21}+P_{23}\right) P_{52}}, \\
& \hat{\alpha}=a+\mathrm{i} \alpha=\frac{\hat{\Theta}_{12}\left(1+P_{23} / P_{31}\right)+\hat{\Theta}_{21}-\hat{\Theta}_{34} P_{23} / P_{31}}{v\left(P_{21}+P_{23}\right)}-\sigma\left(1-\mu_{21}\right)+\frac{B_{25} I_{\mathrm{p}}\left(\hat{\Theta}_{12} / v P_{52}-\sigma_{\mathrm{p}}\left(1-\mu_{52}\right)\right)}{\left(1+B_{52} I_{\mathrm{p}} / v P_{52}\right) v\left(P_{21}+P_{23}\right)},
\end{aligned}
$$




$$
\begin{aligned}
\hat{\beta}=b+\mathrm{i} \beta= & \frac{\left[B_{34}\left(\hat{\Theta}_{12}+\hat{\Theta}_{43}\right)+B_{43}\left(\hat{\Theta}_{12}-\hat{\Theta}_{34}\right)\right] P_{23} / P_{31}+B_{43}\left(\hat{\Theta}_{12}+\hat{\Theta}_{21}\right)}{\nu^{2} P_{43}\left(P_{21}+P_{23}\right)} \\
& -\sigma\left[\frac{B_{43}\left(1-\mu_{21}\right)}{\nu P_{43}}+\frac{B_{34}\left(1-\mu_{43}\right) P_{23}}{v\left(P_{21}+P_{23}\right) P_{31}}\right]+\frac{B_{25} B_{43} I_{\mathrm{p}}\left(\hat{\Theta}_{12} / v P_{52}-\sigma_{\mathrm{p}}\left(1-\mu_{52}\right)\right)}{\left(1+B_{52} I_{\mathrm{p}} / v P_{52}\right) v^{2}\left(P_{21}+P_{23}\right) P_{43}} .
\end{aligned}
$$

As would be expected for insignificant population of the triplet level ( $P_{23} \simeq 0$ ), Eqs. (6)-(9) are transformed to the form (3), (4) for a three-level medium model. An analysis of (1), taking into account (6), shows that the interferometer transmission is at the maximum for zero phase detuning of the interferometer out of resonance:

$$
\Phi=2 \pi n L / \lambda-m \pi=\Phi_{0}+\frac{k_{0} L}{2}\left(\frac{\Theta_{12}}{B_{12}}-\frac{a I+b I^{2}}{1+j I+\beta I^{2}}\right)=0 .
$$

Eq. (10) is a quadratic equation for the signal beam intensity. Two intensity values, for which the interferometer transmission is at the maximum, are associated with two possibilities to realize optical bistability through changes in the refractive index by the transitions in both singlet and triplet channels. From analysis of (10) it follows that optical bistability in both channels may be observed only for a particular relationship between the spectral parameters $a(b / \beta-a / j)<0$. Actually, this inequality means that the nonlinear refractive index is nonmonotonically dependent on the intensity, that is the case for the concurrent contributions of transitions in the principal and excited channels to the medium phase response [8]. In this case increase of the intensity for a specific choice of the interferometer initial detuning out of resonance $\Phi_{0}$ allows for double passage through the transmission maximum of the interferometer - a requisite for realization of optical multistability.

The above situation is illustrated in Fig. 3 giving the interferometer transfer functions which were found for different values of the optical-pumping intensity $I_{\mathrm{p}}$. The following parameters have been selected with the aim of showing the optical multistability mode: the radiation frequency detuning from the absorption profile center equals 0.2 of the profile halfwidth $\left(\eta=\left(\omega-\omega_{12}\right) / \Delta_{12}=0.2\right)$; the band of absorption from the metastable level $T_{1}$ is shifted by two halfwidths to the long-wavelength region of the spectrum related to $\omega_{12}\left(\delta=\left(\omega_{34}-\omega_{12}\right) / \Delta_{12}=-2\right)$; the optical-pumping frequency is tuned out of the center of the absorption band from the excited level $S_{1}$ by 1.6 of the profile halfwidth $\left(\eta_{\mathrm{p}}=\left(\omega_{\mathrm{p}}-\right.\right.$ $\left.\omega_{25}\right) / \Delta_{25}=1.6$ ); the optical density of the medium is $k_{0} L=1$; the initial detuning of the interferometer out of resonance $\Phi_{0}=0.09$. The probabilities of spontaneous and radiationless transitions between different energy states are chosen as follows: $P_{31} / P_{21}=0.078, P_{23} / P_{21}=0.65, P_{43} / P_{21}=2.2, P_{52} / P_{21}=1.1$. A particular arbitrariness in the selection of the spectroscopic medium characteristics is associated with the fact that complex organic compounds exhibit a great variety of characteristics which are largely dependent on the composition of the molecule solvation shell. The intensities of the waves $I_{0}, I_{\text {out }}, I_{\mathrm{p}}$ are given with the normalization factor $B_{12}^{m} / v\left(P_{21}+P_{23}\right)$ determining the saturation intensity of the resonance transition.

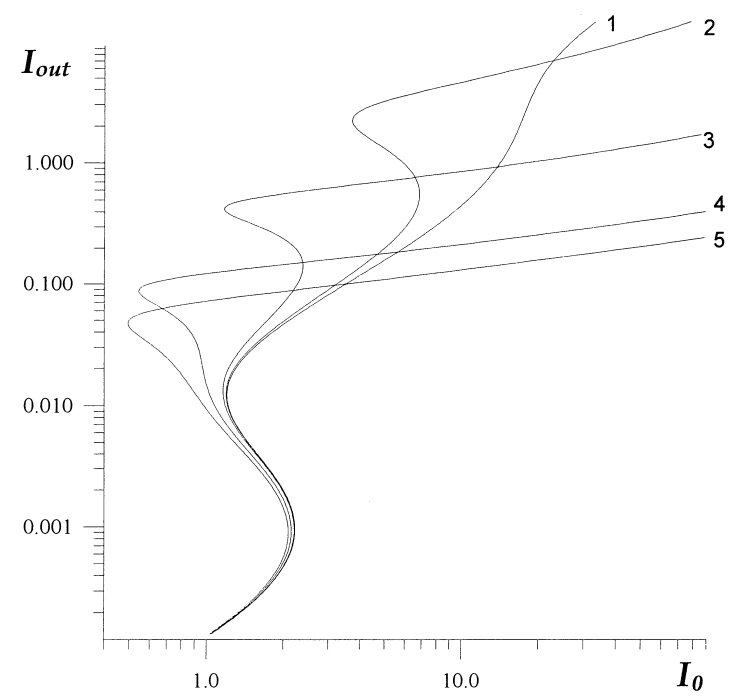

Fig. 3. Optical multistability mode in the interferometer with a five-level resonant medium for different pumping intensities $I_{\mathrm{p}}=0(1), 0.2$ (2), 1 (3), 5 (4), 10 (5). 
As can be seen from Fig. 3, the use of optical pumping results in a new bistable region on retention of the available bistable part of the curve. The positions of the bistable loops are determined by the intensity values characteristic for bleaching of the singlet and triplet channels. The population of the triplet level decreases with the intensity of optical pumping and the developed loop of optical bistability is shifted to lower intensities. Depending on the pumping intensity, double bistability (curve $2, I_{\mathrm{p}}=0.2$ ) or multistability (curve $3, I_{\mathrm{p}}=1$ ) occurs. At large values of the pumping intensity the regions of bistability associated with transitions in the singlet and triplet channels merge (curves 4,5$)$.

\section{Self-oscillations and control over the dynamics of a nonlinear interferometer}

A theoretical analysis of the FPI dynamic modes has been performed based on the five-level resonant-medium model allowing for transitions to the excited singlet and triplet states (Fig. 1c). Such a model has the advantage of four degrees of freedom (owing to the population of different energy levels) making it possible to realize complex dynamic operating modes of the interferometer. It is assumed that the dynamic behavior of the system is defined by the kinetic equations for the level populations, and the output intensity of the interferometer adiabatically follows the changes in the medium parameters. Such a situation is the case for the characteristic time scale of changes in level populations which is far in excess of the setup time of the quasi-stationary field structure in FPI with multiple round-trips of the resonator. Eqs. (1), (2) for a stationary interferometer have been used with the aim of determining the intensity of transmitted radiation under the assumption that the nonstationary character of the interaction is due to the temporal dependence of the nonlinear-layer transmission $\tau$ and phase detuning of light waves $\Phi$. Without allowance for thermal nonlinearity, transmission of a nonlinear layer and phase detuning of FPI out of resonance are determined by the ground and excited states populations and are of the form:

$$
\begin{aligned}
& \tau=\exp \left[-(\hbar \omega L / v)\left(N_{1} B_{12}-N_{2} B_{21}+N_{3} B_{34}-N_{4} B_{43}\right)\right], \\
& \Phi=\Phi_{0}+(\hbar \omega L / 2 v)\left(N_{1} \Theta_{12}-N_{2} \Theta_{21}+N_{3} \Theta_{34}-N_{4} \Theta_{43}\right) .
\end{aligned}
$$

The populations of the energy levels are in turn found from the kinetic equations:

$$
\begin{aligned}
& \partial N_{1} / \partial t=-N_{1} B_{12} U+N_{2} B_{21} U+N_{2} P_{21}+N_{3} P_{31}, \\
& \partial N_{2} / \partial t=N_{1} B_{12} U-N_{2} B_{21} U-N_{2} P_{21}-N_{2} P_{23}-N_{2} B_{25} U_{\mathrm{p}}+N_{5} B_{52} U_{\mathrm{p}}+N_{5} P_{52}, \\
& \partial N_{3} / \partial t=N_{2} P_{23}-N_{3} P_{31}-N_{3} B_{34} U+N_{4} B_{43} U+N_{4} P_{43}, \\
& \partial N_{4} / \partial t=N_{3} B_{34} U-N_{4} B_{43} U-N_{4} P_{43}, \\
& N=N_{1}+N_{2}+N_{3}+N_{4}+N_{5},
\end{aligned}
$$

where $U=I / v, U_{\mathrm{p}}=I_{\mathrm{p}} / v$ are the effective values of the energy volume density of the signal wave and optical pumping in FPI.

Solution of Eqs. (1), (2) taking into account (11)-(13) makes it possible to describe the dynamics of a nonlinear interferometer. A standard linearization procedure of the coupled kinetic equations can be used for determining the conditions of output intensity stability and characteristics of FPI dynamic modes (see Appendix A). Based on such an analysis, the conditions of transition to regular intensity pulsations (Hopf bifurcation) have been determined. At the point of bifurcation the eigenvalues are imaginary and determine the frequency of intensity pulsations of the output radiation. As can be seen from numerical analysis, the frequency of the pulsations is greatly dependent on the intensity of the control beam. Fig. 4 illustrates the calculations performed practically with the same parameters of FPI as in Fig. 3 but with other relative positions of the absorption bands in excited spectral channels and other initial detuning of the interferometer out of resonance. The choice of parameters is dictated by the need for the stability loss of stationary solutions. As an illustration, for demonstrating Hopf bifurcations the signal wave frequency $\omega$ was chosen with a shift relative to the absorption profile maximum $\omega_{12}$ of 2.2 of the profile halfwidth $(\eta=2.2)$; the absorption band from the metastable level $T_{1}$ was shifted by $\delta=2.5$; the frequency of optical pumping was tuned to the center of the absorption band from the excited level $S_{1}\left(\eta_{\mathrm{p}}=0\right)$; the probability of spontaneous and radiationless transitions was $P_{52} / P_{21}=200$; the initial detuning of the interferometer out of resonance was $\Phi_{0}=0.074$.

It can be seen that optical pumping allows for transition from a stationary mode with a constant output intensity (curve 1) to the self-oscillatory mode (curves 2,3 ). The intensity oscillations are conditioned by the changes in transmission $\tau$ (11) and phase detuning of the interferometer $\Phi$ (12) upon transitions of molecules into different excited states. To illustrate the cause of pulsations, Fig. 5 gives the transmission $\tau$, phase detuning $\Phi$ and level populations $N_{i}$ as a function of time. As can be seen, the initial detuning of the interferometer out of resonance has been selected so that the population of the excited $S_{1}$ level (e.g. for $t\left(P_{21}+P_{23}\right) \simeq 34$ ) causes a change in the interferometer state to maximum transmission $(\Phi \simeq 0, \tau \simeq 1)$. Then, owing to intercombination transitions, molecules are relaxed to the metastable level $T_{1}$. As this takes place, the medium 


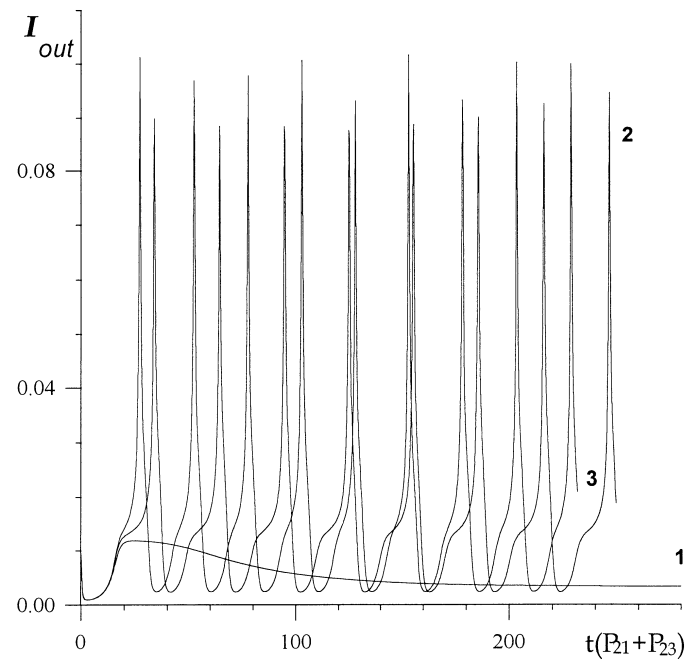

Fig. 4. Oscillations of the output radiation $I_{\text {out }}$ at constant input intensity $I_{0}=0.5$ and different pumping intensities $I_{\mathrm{p}}=0(1), 5(2), 50(3)$.

parameters are chosen so that the radiation frequency, tuned out of the absorption band $\left(S_{0}-S_{1}\right)$ center, falls within the absorption band $T_{1}-T_{2}(\eta \approx \delta)$. A large value of the induced absorption reduces considerably the nonlinear layer transmission $\tau$ as well as the radiation intensity at the interferometer output $I_{\text {out }}$. At low intensities relaxation of the metastable level $T_{1}$ takes place followed by the recovery of the interferometer initial state. And the cycle is repeated.

The nonlinear system considered is very sensitive to changes of the parameters of an intracavity layer. Activation of a small fraction of the molecules with optical pumping to the excited state $S_{2}\left(N_{5} / N \sim 1 \%\right)$ makes it possible to change significantly the dynamic characteristics of the interferometer. In the generation mode the control light beam makes it possible to change both the frequency and form of the oscillations, and to realize transitions between decaying and regular intensity pulsations. Besides, some of the originating oscillations are complex in form. In Fig. 6 the dynamics of changes in
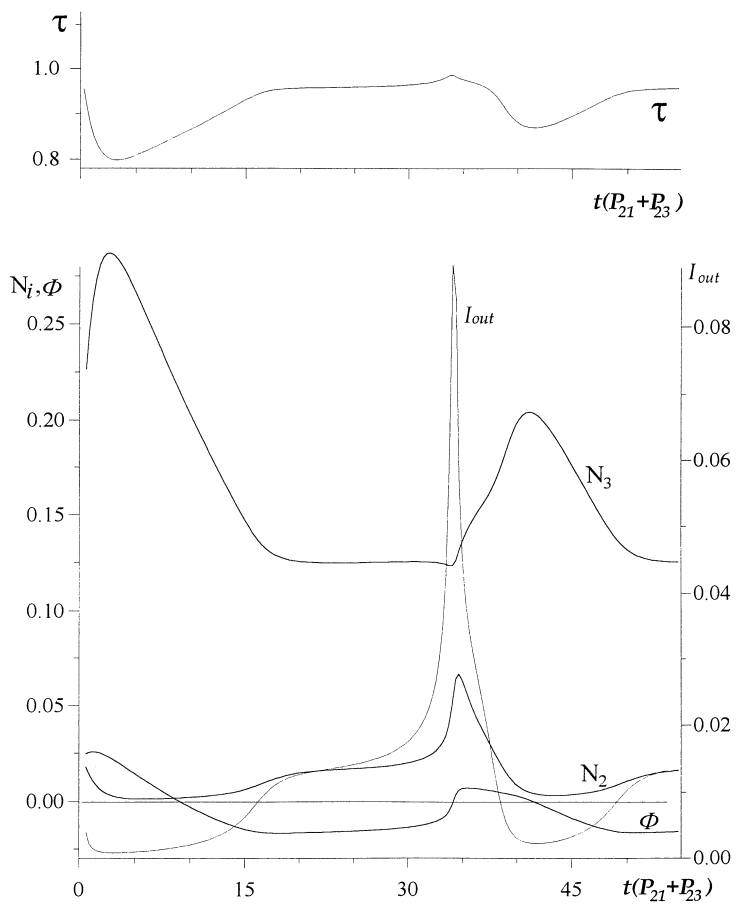

Fig. 5. Kinetic dependences of the resonant medium parameters associated with the self-oscillation mode (Fig. 4, curve 2). 

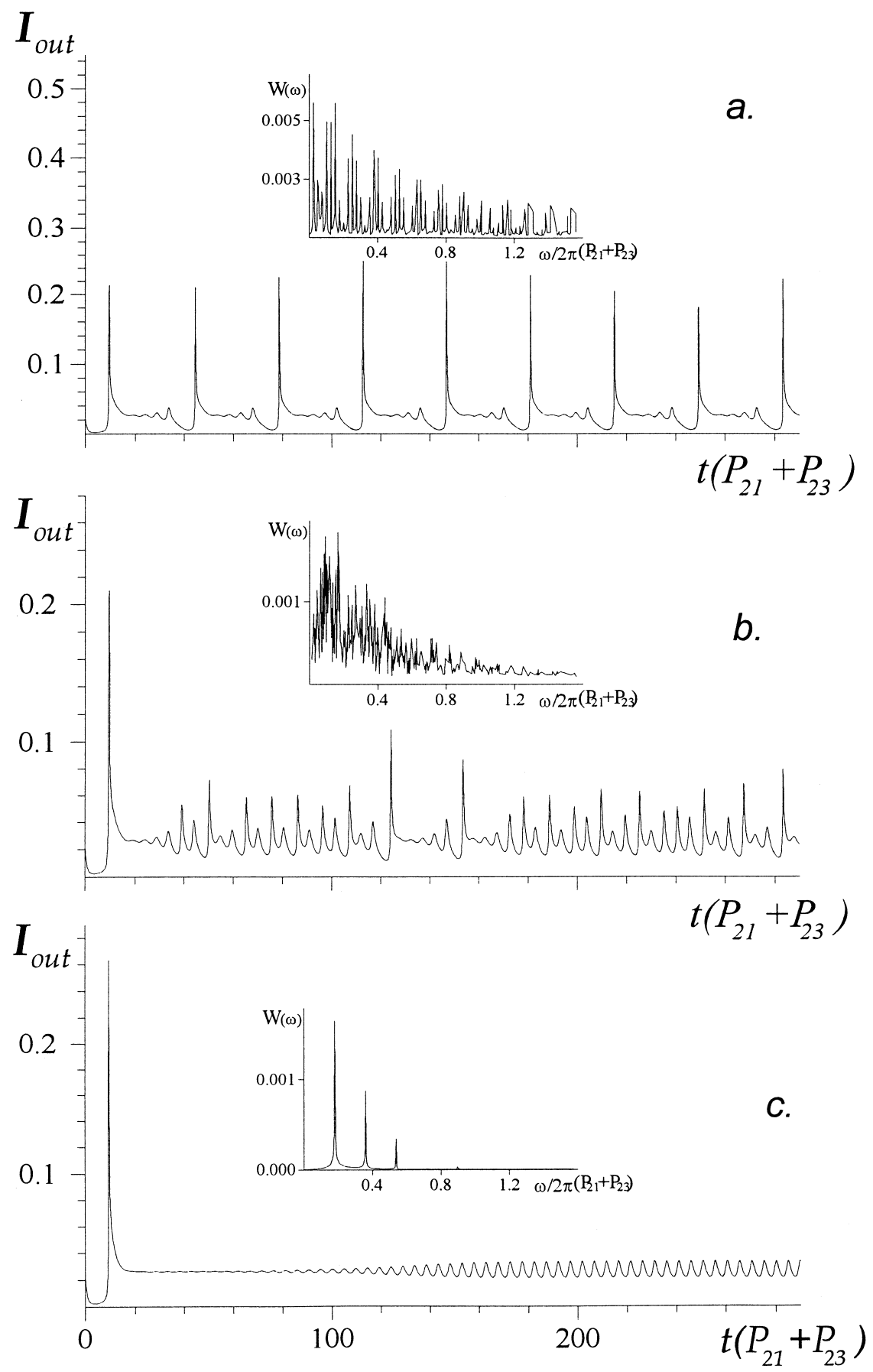

Fig. 6. Complex dynamics of the interferometer for the input intensity $I_{0}=0.935$ and different pumping intensities $I_{\mathrm{p}}=0(\mathrm{a}), 4$ (b), 60 (c), and associated FFT power spectra.

radiation at the output of the interferometer $I_{\text {out }}$ is given as a function of different pumping intensities $I_{\mathrm{p}}$ at constant input intensity $I_{0}$. As opposed to Fig. 4, the changes have been made in the probabilities of transitions $\left(P_{52} / P_{21}=1.1\right)$, phase detuning of the interferometer out of resonance $\left(\Phi_{0}=0.06\right)$ and detuning of the optical-pumping frequency $\left(\eta_{\mathrm{p}}=2.2\right)$. The initial state of the interferometer was associated with the upper branch of the bistable function $I_{\text {out }}\left(I_{0}\right)$. For such a state to be realized, the initial pulse amplification of the input intensity had to be larger than the values of switch-on intensity. Fig. 6 
gives also the fast Fourier transform (FFT) power spectra associated with the steady intensity-pulsation mode. As can be seen, Fig. 6 shows the discrete FFT power spectra peculiar to the periodic vibration modes alongside with the continuous spectra specifying the transition to the mode of optical chaos. The calculated functions may be interpreted proceeding from the analysis of the eigenvalues of the coupled kinetic equations (see Appendix A). Thus, for $I_{\mathrm{p}}=0$ (Fig. 6a) we have $\operatorname{Re} \lambda_{1,2}=0.435, \lambda_{3}=-1.07$ (eigenvalues $\lambda_{i}$ are normalized to the total probability of spontaneous and radiationless transitions $P_{21}+P_{23}$ ) determining the Shilnikov attractor mode [13]. For the pumping intensity of $I_{\mathrm{p}}=4$ we have $\operatorname{Re} \lambda_{1,2}=0.412, \lambda_{3}=-0.608$. The sum of the eigenvalues is larger than zero $\left(\operatorname{Re} \lambda_{1}+\operatorname{Re} \lambda_{2}+\lambda_{3}=0.216\right)$, a requisite for the realization of chaotic pulsations (Fig. 6b). It should be noted that the fourth eigenvalue is a large negative number $\left(\lambda_{4}=-1.19\right)$ and determines a global solution stability. And transition to the self-pulsation mode (Fig. 6c) takes place at $I_{\mathrm{p}}=60\left(\operatorname{Re} \lambda_{1,2}=0.17, \lambda_{3}=-0.371, \lambda_{4}=-2.25\right)$.

Thus, the above analysis demonstrates the possibility of realizing chaotic and regular intensity pulsations in a Fabry-Perot interferometer with resonance nonlinearity, and shows the efficiency of control over the FPI dynamic modes with the use of independent optical pumping.

\section{Conclusion}

This work presents the possibility of controlling optical bistability and dynamic operation modes of the interferometer owing to the change in the nonlinear properties of the intracavity medium upon absorption of an independent light beam (optical pumping) by molecules in the excited energy states. The use of radiation at the frequency of the absorption band from the excited singlet (triplet) level makes it possible to realize incoherent control of FPI transmission, to extend the range of the parameters, for which different operating modes are possible (differential amplification, optical bistability, generation of light-pulses having different forms).

\section{Acknowledgements}

This work was supported partly by the International Soros Science Education Program (ISSEP).

\section{Appendix A}

An analysis of stability for the interferometer states is carried out with the use of the linearization procedure for a system of kinetic equations (13) by small deviations of the energy-level populations from the stationary values $\Delta N_{i}=N_{i}-N_{i}^{\text {st }}$. In the process account must be taken of the fact that the intensity values of a light field in the interferometer (involved in Eqs. (13)) are in their turn dependent on the nonlinear medium transition $\tau$ and phase detuning of light fields $\Phi$ (1). The transformations carried out in conformity with this procedure result in the following system of matrix equations:

$$
\partial \Delta \vec{N} / \partial t=\left\|A_{k l}\right\| \Delta \vec{N}
$$

where $\Delta \vec{N}$ is the vector composed of $\Delta N_{i}$ elements, $\left\|A_{k l}\right\|$ is a matrix, which elements are governed by the following relations:

$$
\begin{aligned}
& A_{11}=\left(P_{21}+P_{23}\right) \Psi_{1}-B_{12} I / \nu, \\
& A_{12}=\left(P_{21}+P_{23}\right) \Psi_{2}+B_{21} I / \nu+P_{21}, \\
& A_{13}=\left(P_{21}+P_{23}\right) \Psi_{3}+P_{31}, \\
& A_{14}=\left(P_{21}+P_{23}\right) \Psi_{4}, \\
& A_{21}=-\left(P_{21}+P_{23}\right) \Psi_{1}+B_{12} I / \nu-B_{52} I_{\mathrm{p}} / \nu-P_{52},
\end{aligned}
$$




$$
\begin{aligned}
& A_{22}=-\left(P_{21}+P_{23}\right)\left(\Psi_{2}+1\right)-B_{21} I / \nu-\left(B_{25}+B_{52}\right) I_{\mathrm{p}} / \nu-P_{52} \\
& A_{23}=-\left(P_{21}+P_{23}\right) \Psi_{3}-B_{52} I_{\mathrm{p}} / \nu-P_{52} \\
& A_{24}=-\left(P_{21}+P_{23}\right) \Psi_{4}-B_{52} I_{\mathrm{p}} / \nu-P_{52} \\
& A_{31}=\left(P_{21}+P_{23}\right) \Psi_{1} \xi=-A_{41} \\
& A_{32}=\left(P_{21}+P_{23}\right) \Psi_{2} \xi+P_{23}=-A_{42}+P_{23} \\
& A_{33}=\left(P_{21}+P_{23}\right) \Psi_{3} \xi-P_{31}-B_{34} I / \nu=-A_{43}-P_{31} \\
& A_{34}=\left(P_{21}+P_{23}\right) \Psi_{4} \xi+P_{43}+B_{43} I / \nu=-A_{44}
\end{aligned}
$$

where

$$
\begin{aligned}
& \Psi_{1}=(N \hbar \omega L / v)\left[B_{12} \Omega_{1}+\Theta_{12} 2 R \sin 2 \Phi\right] \Omega_{2}, \\
& \Psi_{2}=(N \hbar \omega L / v)\left[-B_{21} \Omega_{1}-\Theta_{21} 2 R \sin 2 \Phi\right] \Omega_{2}, \\
& \Psi_{3}=(N \hbar \omega L / v)\left[B_{34} \Omega_{1}+\Theta_{34} 2 R \sin 2 \Phi\right] \Omega_{2}, \\
& \Psi_{4}=(N \hbar \omega L / v)\left[-B_{43} \Omega_{1}-\Theta_{43} 2 R \sin 2 \Phi\right] \Omega_{2}, \\
& \xi=\left(B_{34}+B_{43}^{2} I / \nu P_{43}\right) I /\left(B_{43} I+\nu P_{43}\right), \\
& \Omega_{1}=\left(1-\tau^{2} R^{2}\right) / \tau+I_{0}(1-R)\left[(1-\tau)(1+\tau R) /(k L)-1-\tau^{2} R\right] /(I \tau k L), \\
& \Omega_{2}=B_{12} I^{2}\left(1+B_{43} I / \nu P_{43}\right) /\left[I_{0}\left(1+j I+\beta I^{2}\right) v\left(P_{21}+P_{23}\right)\right] .
\end{aligned}
$$

The characteristic equation that defined the eigenvalues of the linearized coupled equations could be written as follows:

$$
\lambda^{4}+\xi_{1} \lambda^{3}+\xi_{2} \lambda^{2}+\xi_{3} \lambda+\xi_{4}=0,
$$

where

$$
\begin{aligned}
& \xi_{1}=-\zeta_{1}-\left(\zeta_{7}+\zeta_{3}\right) P_{23} /\left(P_{21}+P_{23}\right) \\
& \xi_{2}=-\zeta_{2}-\left(\zeta_{1} \zeta_{7}-\zeta_{8}-\zeta_{4}-\zeta_{3} \zeta_{5}\right) P_{23} /\left(P_{21}+P_{23}\right) \\
& \xi_{3}=\left(\zeta_{2} \zeta_{7}+\zeta_{1} \zeta_{8}-\zeta_{4} \zeta_{5}-\zeta_{3} \zeta_{8}\right) P_{23} /\left(P_{21}+P_{23}\right) \\
& \xi_{4}=\left(\zeta_{2} \zeta_{8}-\zeta_{4} \zeta_{6}\right) P_{23} /\left(P_{21}+P_{23}\right) \\
& \zeta_{1}=A_{11}-A_{12}-\left(A_{13}-A_{14}\right) P_{23} / P_{31}-\left(B_{25}+B_{52}\right) I_{\mathrm{p}} / v-P_{52}, \\
& \zeta_{2}=\left(A_{11}-A_{12}-\left(A_{13}-A_{14}\right) P_{23} / P_{31}\right)\left(B_{52} I_{\mathrm{p}} / v+P_{52}\right)-A_{11} B_{25} I_{\mathrm{p}} / v \\
& \zeta_{3}=\left(A_{24}-A_{23}\right)\left(P_{21}+P_{23}\right) / P_{31}, \\
& \zeta_{4}=\left(A_{11}-A_{14}\right)\left(P_{21}+P_{23}\right) \\
& \zeta_{5}=\left(A_{31}-A_{32}\right) P_{31} / P_{23}+P_{31}+A_{43}-A_{44}+B_{52} I_{\mathrm{p}} / v+P_{52}, \\
& \zeta_{6}=\left[\left(A_{31}-A_{32}\right) P_{31} / P_{23}+P_{31}+A_{43}-A_{44}\right]\left(B_{52} I_{\mathrm{p}} / v+P_{52}\right)-A_{41} B_{25} I_{\mathrm{p}} P_{31} / v\left(P_{21}+P_{23}\right) \\
& \zeta_{7}=\left(A_{44}-A_{43}\right)\left(P_{21}+P_{23}\right) / P_{23}+\left(P_{21}+P_{23}\right) P_{31} / P_{23}, \\
& \zeta_{8}=\left(A_{31}-A_{34}\right)\left(P_{21}+P_{23}\right) P_{31} / P_{23} .
\end{aligned}
$$

As can be seen, the characteristic equation is a fourth-order equation and may have a classical solution, assuming construction of the cubic resolvent and using the Cardano formulas for solution of the cubic equation.

\section{References}

[1] H.M. Gibbs, Optical Bistability: Controlling Light with Light, Academic Press, Orlando, 1985.

[2] N.M. Lavandy, W.S. Rabinovich, IEEE J. Quantum Electron. 20 (1984) 458.

[3] I.N. Agishev, S.M. Karpuk, A.S. Rubanov, A.L. Tolstik, Pis'ma Zh. Tekh. Fiz. 20 (1994) 28, in Russian; Sov. J. Technol. Phys. Lett. 20 (1994) 619.

[4] M. Haelterman, P. Mandel, J. Danckaert, H. Thienpont, I. Veretennicoff, Optics Comm. 74 (1989) 238. 
[5] J.L. Jewell, H.M. Gibbs, S.S. Tarng, A.C. Gossard, W. Wiegmann, Appl. Phys. Lett. 40 (1982) 291.

[6] N.A. Ivanova, A.S. Rubanov, A.L. Tolstik, A.V. Chaley, Photonic Switching, Proc. SPIE. 1807 (1992) 147.

[7] N.A. Ivanova, A.S. Rubanov, A.L. Tolstik, A.V. Chaley, Izv. Ross. Akad. Nauk Ser. Fiz. 57 (1993) 149, in Russian.

[8] V.V. Kabanov, A.S. Rubanov, A.L. Tolstik, Kvant. Elektron. 15 (1988) 1681, in Russian; Sov. J. Quantum Electron. 18 (1988) 1047.

[9] D.A.B. Miller, IEEE J. Quantum Electron. 17 (1981) 306.

[10] R.H. Pantell, H.E. Puthoff, Fundamentals of Quantum Electronics, Wiley, New York, 1969.

[11] V.V. Kabanov, A.S. Rubanov, Dokl. Akad. Nauk BSSR 24 (1980) 34, in Russian.

[12] S.M. Karpuck, A.S. Rubanov, A.L. Tolstik, A.V. Chaley, Transverse Patterns in Nonlinear Optics, Proc. SPIE. 1840 (1992) 43.

[13] V.S. Anishchenko, Complicated Oscillations in Simple Systems: Appearance Routes, Structure and Properties of Dynamical Chaos in Radiophysical Systems, Nauka, Moscow, 1990. 